\title{
Estrategias para la recaudación del anticipo del impuesto a la renta en el Ecuador
}

\section{Strategies for the Advance Income Tax Payment in Ecuador}

Cinthia Elizabeth González Valladares. ${ }^{1}$, Cecilia Ivonne Narváez Zurita. ${ }^{2}$ \& Juan Carlos Erazo Álvarez. ${ }^{3}$

DOI: https://doi.org/10.33262/visionariodigital.v3i2.1.547

\begin{abstract}
.
The Organic Code of Planning and Public Finances points out that any measure that affects tax collection must be compensated to assure the sustainability of the general budget of the State. Therefore, through the Internal Tax Regime Law, the advance income tax payment was created. This measure is not minimize the recurring tax avoidance in the Ecuadorian tax culture adapting to its formula factors that include the economic operating of the taxpayers. In this sense, the method to advance tax calculation is determined by the previous tax year regardless of the current economic situation of the taxpayer, since it is taxed from a different tax year which is declaring.

For all these reasons, this article aims to design strategies to collect advance tax payments taking into account the economic situation of the taxpayers during the tax period to be declared. Therefore, a comparative analysis regarding tax control among several Latin American Countries was carried out. Also, the control over avoidance operating was evaluated in the Ecuadorian Tax Administration. All this research was developed using approaches like inductive-deductive and analytical-synthetic. The results show the need to improve the control over the collect advance tax payments, keep tax exemptions to increase the productivity of enterprises, consider the

\footnotetext{
1 Universidad Católica de Cuenca, Posgradista Maestría en Contabilidad y Auditoría, Cuenca, Ecuador, cegonzalezv187@psg.ucacue.edu.ec

2 Universidad Católica de Cuenca, Departamento de Posgrado, Cuenca, Ecuador, inarvaez@ucacue.edu.ec

${ }^{3}$ Universidad Católica de Cuenca, Departamento de Posgrado, Cuenca, Ecuador, jcerazo@ucacue.edu.ec
} 
information during the current year to establish the advance tax payments, and finally train all taxpayers on tax exemption field.

Keywords: Advance income tax payment, exemption, tax collection, tax avoidance, strategies.

\section{Resumen.}

Código Orgánico de Planificación de Finanzas Publicas señala que toda medida que afecte a la recaudación de impuestos deberá ser compensada a fin de que se garantice la sostenibilidad del presupuesto general del Estado, por esta razón, mediante la Ley de Régimen Tributario Interno se creó el anticipo del impuesto a la renta como medida para minimizar la elusión de tributos como elemento recurrente en la cultura tributaria de los ecuatorianos, adaptando en su fórmula factores que incluyen de forma integral la operatividad económica de los contribuyentes. En este sentido, el método de cálculo actual del anticipo del impuesto a la renta es determinado con relación a información del ejercicio impositivo anterior sin considerar la situación económica actual del contribuyente, puesto que se grava al resultado de un ejercicio impositivo distinto al que se está declarando.

Por lo antes expuesto, el presente artículo tiene por objetivo diseñar estrategias para la recaudación del anticipo del impuesto a la renta, considerando la situación económica de los contribuyentes en el periodo impositivo a declarar. Con este fin, se llevó a cabo un análisis comparativo entre varios países de Latinoamérica relacionados con el control fiscal, además se procedió a evaluar en la Administración Tributaria del Ecuador los procesos de control de omisidad por concepto de anticipo del impuesto a la renta, bajo el uso de los métodos inductivo - deductivo y analítico - sintético. Los resultados muestran la necesidad de: mejorar el proceso de control sobre la recaudación del anticipo del impuesto a la renta; conservar las exenciones que incentivan la productividad de las empresas; considerar la información del año en curso para determinar el pago del anticipo del impuesto a la renta y capacitar a los contribuyentes en materia de exenciones.

Palabras claves: anticipo del impuesto a la renta, omisidad, recaudación, evasión y elusión, estrategias.

\section{Introducción.}

Los impuestos son la prestación que se genera al ocurrir un hecho impositivo y que deberían servir para financiar los servicios públicos, dichos impuestos se caracterizan por ser directos e indirectos gravando de esta manera al patrimonio, a la producción, al consumo entre otras 
actividades, dependiendo de las capacidades contributivas se generará la cuantía del impuesto (Loyo, 2003). Los impuestos son tributos que se generan por la existencia de un hecho económico o jurídico a consecuencia de la generación de un patrimonio, de una venta, adquisición o cualquier tipo de evento que permita al sujeto pasivo la generación de un ingreso (González y Lejeune, 2003).

Delgado y Muñiz (2005) refieren que el impuesto es un compromiso sin compensación adquirido por los ciudadanos, que se basa en realizar el pago de ciertos valores que normalmente es representado en dinero y que debe ser a favor del Estado o incluso de otros organismos públicos, dichas prestaciones son fijadas mediante condiciones con carácter de obligatorio y de entrega exclusiva al ente acreedor de dicho impuesto. Dentro del tema tributario el Estado ejerce poder sobre la capacidad de contribución que tienen los sujetos pasivos en base a las leyes expedidas en el país. Troya (como se citó en Benítez 2008) piensa que los impuestos son las retribuciones que exige el Estado basado en su poder de supremacía y que el contribuyente por disposición legal se ve en la obligación de pagar la existencia de dicho gravamen.

Zambrano (2014) indica que los impuestos constituyen para el sujeto pasivo el pago de obligaciones sin que esto obligue al Estado a otorgar algún tipo de retribución o contraprestación de un servicio o beneficio para dicho contribuyente. Es importante indicar que según la normativa tributaria vigente se considera como contribuyente a toda persona natural o jurídica a quien mediante ley se impone una obligación tributaria siempre que se haya suscitado el hecho generador de la obligación, es decir estos serán los responsables directos del pago del pago de los impuestos (Asamblea Nacional Constituyente, 2016).

En base a las definiciones antes citadas se puede evidenciar que los autores consideran que los impuestos que son entregados por los contribuyentes hacia el Estado se caracterizan por ser coercitivos y que no se deben informar sobre la forma en que dichas recaudaciones son retribuidas hacia la ciudadanía en general, pero que sí se debe tener presente que constituyen una herramientas fundamental que permite viabilizar la redistribución equitativa de los ingresos; compartiendo el criterio de González y Lejeune (2003) que parten del punto de vista que todo impuesto debe generarse de una actividad económica pues es la única manera en que podemos garantizar que el pago realizado por los contribuyentes sea efectivo, ya que se origina en base a un hecho económico real que permite la generación de ingresos sea por la compra o venta de un bien o servicio, por tal razón se debe tener presente que al encontrarnos dentro de una sociedad organizada, los sujetos pasivos deben cumplir con las obligaciones y el pago de ciertos valores establecidos mediante normativa legal las mismas deben estar acorde a las condiciones y características de las actividades generadoras del ingreso, procurando que afecte de manera directa a la capacidad de contribución que tiene cada ciudadano sin que esto limite su producción. 


\section{Elementos de la obligación tributaria.}

Según Troya (2008) la obligación tributaria se origina debido a que tanto los ciudadanos y los residentes se benefician directa o indirectamente de las obras y servicios públicos en la sociedad en que transitan, por ello, debe regularse de forma clara cuándo una persona está obligada a contribuir para solventar los gastos públicos. La obligación tributaria dentro del ámbito legal es considerada como el vínculo existente entre el ente acreedor representado por el Estado y el ente deudor que son los contribuyente, del hecho generador normado mediante ley, el compromiso contraído como consecuencia de este vínculo permite satisfacer las deudas contraídas por el Estado, actualmente el SRI es la institución encargada de gestionar el cumplimiento de obligaciones tributarias relacionadas con el ingreso, la transferencia de bienes y servicios, entre otras (Andrade, 2013).

La normativa tributaria contempla que aspectos se debe considerar para la creación de un impuesto, los mismos que parten desde su nacimiento, los elementos que originan la obligación tributaria son el hecho generador del tributo, la base imponible o la determinación de la obligación e incluso los sujetos que intervienen en la misma (figura 1), para el caso nacional el Código Tributario rige el vínculo jurídico que originan los tributos establecidos por parte del Estado, así como también permite identificar los ilícitos tributarios, e indica que la Administración Tributaria tiene la facultad sancionadora por el incumplimiento de las obligaciones por parte de los sujetos pasivos (Torrico, 2015).

Figura 1. Elementos de la obligación tributaria

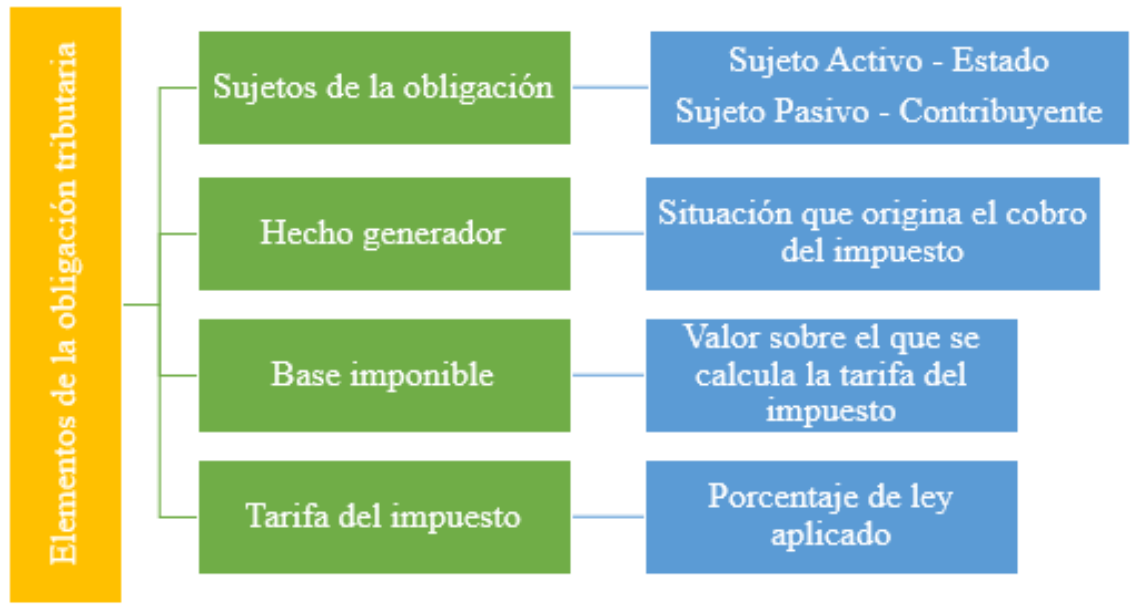

Fuente: Torrico (2015).

El cumplimiento de obligaciones tributarias se ve ligado a la adecuada gestión que se dé a los recursos públicos y de esta manera poder garantizar a la ciudadanía el buen destino de los 
tributos que se cobra, en este sentido las administraciones de turno deben garantizar que los ciudadanos reciban información continua sobre la gestión que se da a los recursos percibidos, permitiendo que se genere confianza entre el Estado y los ciudadanos a fin de promover la comprensión y cumplimiento voluntario de las obligaciones tributarias por parte de la ciudadanía (Procuraduría de la Defensa del Contribuyente, 2015).

Según el Código Tributario una obligación nace cuando se verifica el cumplimiento del hecho generador de un impuesto debiendo satisfacer por ello una prestación en dinero, especies o servicios por parte de los contribuyentes o responsables hacia el Estado en base a la establecido en la ley (Asamblea Nacional Constituyente, 2016). Lapatza (como se citó en Hernández, 2016) menciona que la obligación tributaria se enmarca en el principio de capacidad económica, pues los tributos deben ser establecidos y cobrados por el ente regulador cuando se susciten hechos que directa o indirectamente estén relacionados con la capacidad económica, en este sentido solo deberá exigirse cuando existe capacidad económica motivando que se establece en cierta medida una justicia tributaria.

Dentro de los elementos principales que intervienen al momento de determinar una obligación se deberán considerar los siguientes:

Según la Asamblea Nacional Constituyente (2016) dentro del artículo 16 del Código Tributario establece que el hecho generador es la base imponible sobre la cual se calcula un tributo, por ejemplo, se podría indicar que el hecho generador en una venta es el ingreso que percibe el sujeto pasivo y sobre el cual se tiene que determinar un tributo a favor del Fisco. Salazar (2008) establece que el sujeto activo del impuesto a la renta es toda institución del sector público demandante de un impuesto; es decir, el sujeto activo tiene la potestad de administrar y percibir los tributos o impuestos percibidos de los contribuyentes.

La Asamblea Nacional Constituyente (2016) establece que según el artículo 24 del código tributario se considera como sujeto pasivo a toda persona natural o jurídica que en base a la ley se encuentra obligada a la prestación de una obligación tributaria que puede ser como contribuyente directo o como representante para el caso de sociedades, por lo que será considerada como el ente que realiza la declaración o pago de un impuesto a fin de cumplir lo establecido en la normativa.

Aunque algunos de los autores citados hacen énfasis en que la obligación tributaria nace del vínculo jurídico que existe entre el Estado y los contribuyentes, es importante destacar que comparto con lo citado por el autor Hernández (2016) pues se considera que como condición indispensable para la imposición se debe evaluar la capacidad económica que tengan los contribuyentes, es decir, se debe verificar que se produzca el hecho generador y la base imponible de dicho impuesto permitiendo de esta manera que el sujeto activo pueda establecer una cuantificación económica sobre la cual se establecerá un tipo impositivo, lo 
que a su vez nos permitirá alcanzar una recaudación eficiente por parte del ente de control y dicha contribución permitirá la sostenibilidad del gasto público.

\section{Recaudación del impuesto a la renta y del anticipo.}

Stiglitz (como se citó en Torres, 2008) refiere que un buen sistema tributario debe basarse en escenarios relacionados a la eficiencia, flexibilidad y en la equidad de las economías. El sistema tributario en el Ecuador debe interferir lo menos posible sobre la asignación de recursos y debe estar enfocada en la recaudación de ingresos al menor costo posible para los sujetos pasivos, lo que se debe tener presente es que a mayores impuestos se puede incidir en el incentivo al trabajo y el ahorro por parte de los ciudadanos y esto va a repercutir de manera directa en la economía del país.

Un régimen tributario debe ser claro y sólido de esta manera fomentará la inversión tanto nacional como internacional y por otro lado favorecerá a los contribuyentes al brindarles confianza y estabilidad, en base a esto se define que el sistema tributario debe estar enmarcado en el principio de irretroactividad de la norma el que indica que no se podrán dictar leyes si estas causaren perjuicio a los contribuyentes, procurando la transparencia de las disposiciones legales (Rodas, 2006).

Vergara (2011) afirma que los sistemas tributarios son el pilar fundamental de las economías de los países ya que a través de la recaudación de impuestos se generan ingresos en base a factores relacionados a la capacidad para fomentar actividades productivas y también de la voluntad de cumplir con el pago de impuestos por parte de las empresas, un sistema tributario debe estar apoyado en leyes fiscales que permitan generar servicios para los ciudadanos, y logar de esta manera que los contribuyentes por cuenta propia determinen sus responsabilidad del pago correcto de impuestos, sin dejar de lado que para lograr este objetivo se debe establecer normas claras, sencillas y de fácil comprensión para los contribuyentes.

Doing Business (como se citó en Vergara, 2011) indica que cuando existen sistemas de impuestos complejos generan un mayor riesgo de evasión, en este caso tienden a haber mayores sectores informales, por lo que si se estructura un sistema tributario que sea útil y de fácil comprensión para los contribuyentes esto fomentará el desarrollo del sector privado y la formalización e inversión en nuevas empresas generando empleo y crecimiento económico.

En varios países existen diferencias entre la aplicación de un sistema tributario o un régimen tributario, considerando que se debe normar en base a los objetivos plantados según las características jurídicas, económicas y sociales por cada territorio, en el Ecuador se ha establecido un régimen tributario, pero en otros territorios andinos sus administraciones se argumentan en sistemas tributarios. Un sistema tributario se caracteriza por una correcta integración de los tributos, en este caso se establecen pocos impuestos pero se respaldan en 
la representatividad de los mismos en cuanto a capacidad contributiva, teniendo procesos sencillos tanto para el contribuyente como para la administración, por otro lado un régimen tributario es menos exigente y se basa en varios principios como los de progresividad, generalidad, equidad entre otros y está acorde a condiciones pocos rigurosas en donde la implementación de un impuesto busca una imposición confiscatoria de la economía (Chuquimarca, 2013).

La Asamblea Nacional Constituyente (2015) afirma que un sistema tributario debe caracterizarse por cumplir ciertas condiciones tales como eficiencia económica, flexibilidad y equidad, procurando que la distribución de la carga impositiva se respalde en impuestos que permitan disminuir la desigualdad y procuren el logro de justicia social, también el sistema tributario debe basarse en el principio de progresividad con el que se busca la mayor recaudación por parte de los impuestos directos a fin de que se afecte a la capacidad económica que tienen los individuos, todo esto en base a un marco legal necesario para el manejo eficiente de tributos equitativos.

\section{Antecedentes del impuesto a la renta.}

Andino (como se citó en Arias, 2009) afirma que en los países de América Latina el impuesto a la renta fue establecido durante los años 20 , pero en los años 50 se consolido como parte principal del sistema tributario siendo para los gobiernos el pilar fundamental para la recaudación de ingresos hasta la llegada de otro tipo de impuestos. El sistema tributario se encarga de recaudar fondos que constituyen la principal fuente de ingresos para el Estado y que son destinados a cubrir el gasto social, estos fondos son obtenidos mediante la formulación de un grupo de impuestos estructurados en base a políticas que buscan una distribución equitativa de los ingresos que obtiene la población, así como fomentar el aparato productivo y regular el consumo.

En el Ecuador el impuesto a la renta se origina en el año 1925 en base a un plan de modernización de las finanzas públicas y privadas del profesor Edwin Kemmerer y fue aprobado en el año de 1926 mismo que grava de manera separada a las rentas que provienen del trabajo o servicio y a las rentas que provienen del capital puesto que se consideraba un impuesto justo pues se basaba en el principio de capacidad de pago (ibídem).

Andino (como se citó en Ramírez, Cano y Oliva, 2009) refiere que en el año de 1945 fue creado el impuesto a las ganancias excesivas con lo que surge la definición de renta global, concepto que busca unificar todas las rentas percibidas por los contribuyentes, es decir agrupó a todos los impuestos que gravaban a las rentas locales y nacionales tanto de capital como de trabajo.

En el año 2007 se realizaron cambios al Impuesto a la renta mismo que fueron regulados en la Ley de régimen tributario Interno, estos cambios consistían en establecer una tabla 
progresiva que iba entre el $25 \%$ y $35 \%$ del impuesto en base a rangos imponibles, adicionalmente se incluyó la deducción por gastos personales y la exoneración de los ingresos percibidos por décimo tercera y cuarta remuneración por parte del sujeto pasivo, para el caso de las personas con discapacidad y de la tercera edad podían deducirse de su impuesto a la renta hasta el 50\% del ingreso gravado o hasta dos y tres veces la fracción básica exenta (Ramírez et al., 2009).

Salazar (2008) refiere que actualmente en el Ecuador el Impuesto a la renta es uno de los principales impuestos que es controlado por parte de la Administración Tributaria, pues desde la historia este impuesto gravaba a las ganancias obtenidas por las unidades económicas durante un ejercicio, hoy por hoy representa el impuesto de mayor recaudación de las arcas fiscales y que permite contribuir en gran medida al desarrollo de los programas establecidos por el Estado.

En la mayor parte de las legislaciones de América Latina se encuentran establecido el impuesto a la renta en base al tipo de contribuyente, debiendo diferenciarlo según la carga tributaria que estos generen, por ello se definen dos formas de imposición tanto para las personas naturales como para las personas jurídicas. Este impuesto tiene diferentes denominaciones por lo que es necesario identificarlas según el tipo de sistema o régimen tributario, esto permitirá la comprensión de los términos al momento del estudio, por lo que serán citadas en la siguiente tabla (Arias y Cano, 2009).

Asamblea Nacional Constituyente (2007) establece como objeto del impuesto a la renta al tributo que grava a las ganancias totales de las sociedades dentro del territorio nacional o extranjeras con domicilio local, así como también los ingresos luego de deducciones establecidas en la ley para personas naturales y para las sucesiones indivisas o herederos con derechos a renta de acuerdo a lo dispuesto en la Ley de Régimen Tributario Interno, además en el artículo 2 de la presente ley se define el concepto de renta como los ingresos de origen nacional obtenidos producto del trabajo, de rendimientos del capital, o de ambas fuentes, sean estos pagados en dinero, especies o servicios y a los ingresos conseguidos fuera del territorio nacional por personas naturales con domicilio en el Ecuador o por sociedades nacionales con personería jurídica contempladas en su artículo 98.

La denominación de renta o ingreso para el caso de una persona se relaciona con las ganancias totales que recibe durante un periodo fiscal, debiendo considerar que dichas ganancias podrán ser destinadas tanto al consumo como al ahorro, lo que va a generar un aumento del patrimonio del sujeto pasivo, el flujo de dicho impuesto puede ser medido por la fuente generadora del mismo o por el destino que se les dará a los fondos percibidos, se debe tener presente que las normas tributarias definen la base imponible sobre la cual se deberá gravar y también identificará los componentes de las ganancias que no serán gravados (Piffano, 2012). 
Fisher (como se citó en Noya, et al., 2014) afirma que según su teoría del incremento neto patrimonial se considera renta a todo ingreso por transacciones, bienes y retribución que sea percibida por parte del contribuyente asumiendo la teoría del incremento patrimonial, no obstante, señala que no debe gravarse el ahorro. Renta, según el autor, es todo flujo de servicios que disfruta el contribuyente, los bienes que posee y su retribución, incluidas las rentas psíquicas. Esta posición ha sido criticada por su inaplicabilidad, con el argumento que dicho impuesto dejaría de ser un impuesto a la renta, para pasar a ser un impuesto suntuario al gasto.

El impuesto a la renta grava a las ganancias producidas por una inversión, a los ingresos que sean producto del trabajo bajo relación de dependencia, por las actividades comerciales o profesionales u otras actividades que produzcan rentabilidad, por este motivo en el Ecuador se considera al impuesto a la renta como un impuesto directo que permite recaudar recursos de los contribuyentes mediante la imposición de una tarifa sobre las riquezas que se generan producto de las actividades económicas y que permiten de esta manera financiar los gastos del Estado (Mejía, 2015).

Si bien el impuesto a la renta es considerado por los autores antes citados como uno de los principales impuestos en los sistemas o regímenes tributarios y que se basa en una imposición tributaria a los ingresos percibidos por los contribuyentes que generen una actividad económica, se debe mencionar que el autor Mejía (2015) considera al impuesto a la renta como un impuesto directo pues grava al renta proveniente del capital o del trabajo y esto permite que la redistribución de la riqueza, debemos tener presente que en la actualidad en el Ecuador se está mejorando la estructura tributaria procurando que los ingresos percibidos por las cargas tributarias sean los más equitativos posibles y que sean regulados a fin de que se reduzcan los procesos inflacionarios y que en cierto momento se pueda recuperar la confianza de la ciudadanía para motivar a la inversión en el país.

\section{Generalidades del anticipo del impuesto a la renta.}

La composición de los ingresos tributarios se basa en diferentes teorías principalmente en la de eficiencia donde el impuesto podrá estimular o disminuir de manera general el bienestar de los contribuyentes y el de equidad verificando si la creación de un impuesto es justo para todos, en este caso el impuesto sobre la renta se relaciona sobre ambas teorías incluyendo un costo adicional del bienestar determinado por la eficiencia ya que al momento de gravar a la renta representa un costo más elevado debido a que es un impuesto establecido sobre el trabajo y capital disminuyendo la capacidad de ahorro por parte de los sujetos obligados a tributar (Tanzi y Zee, 2001).

El anticipo del impuesto a la renta permite al Estado recibir por adelantado una parte del impuesto antes de su vencimiento, exigibilidad o cobro, desde el punto de vista económico generalmente la determinación y pago de este impuesto puede ser beneficioso o perjudicial 
para el contribuyente puesto que el desembolso de dicha obligación no se realizará en un solo momento sino el contribuyente deberá realizar varios desembolsos en diferentes tiempos a fin de cubrir el pago de dicho impuesto (Mejía, 2015).

El Centro de Estudios Fiscales (2017) define el anticipo del impuesto a la renta como el pago realizado por adelantado por parte de los sujetos pasivos de dicho impuesto, a fin de prevenir la evasión permitiendo al Estado mantener un flujo de dinero y de esta manera disminuir el impacto negativo que puede provocarse en la liquidez de los contribuyentes al momento de la recaudación, puesto que dichas cuotas serán disminuidas al momento de hacer el pago del impuesto a la renta global.

Rondón (2017) señala que el anticipo del impuesto a la renta es la obligatoriedad de cumplimiento de un tributo exigible de forma anticipada basada en la proyección de que se genere un impuesto futuro. En esta definición se observa una postura específica de la Administración Tributaria con enfoque en la conceptualización de un tributo generador de ingresos para el Estado, basado en cierto grado de confiabilidad en la estabilidad de una estructura económica dentro de los periodos fiscales en los que se define éste impuesto.

Las constantes reformas a la Ley de Régimen Tributario Interno establecen dos formas de cálculo del anticipo del impuesto a la renta por un lado están direccionadas hacia las sociedades, personas naturales y las sucesiones indivisas obligadas a llevar contabilidad que deberán calcular el anticipo para el próximo ejercicio mediante la suma del $0.4 \%$ de los activos, 0.4 de los ingresos del año en curso, el $0.2 \%$ del patrimonio y el $0.2 \%$ de los gastos; $\mathrm{y}$ por otro lado las personas naturales y sucesiones indivisas no obligadas a llevar contabilidad o las que estén obligadas a llevar contabilidad pero que no realicen actividad económica en el periodo, así como las sociedades y organizaciones de economía popular y solidaria que estén bajo la condición de microempresas, y la empresas que suscriban o tengan suscritos contratos de exploración y explotación de hidrocarburos que se basa en el $50 \%$ del impuesto a la renta determinado en el ejercicio fiscal menos las retenciones del impuesto a la renta que se le hayan efectuado en el mismo periodo (Asamblea Nacional Constituyente, 2017).

\section{Estrategias de control fiscal en los sistemas tributarios nacionales e internacionales}

Se debe iniciar citando la conceptualización de estrategias, para lo que Robbins y Coulter (2005) indican que es importante diseñar estrategias que permitan atraer clientes para lograr que la organización pueda desarrollarse, pues en sí las estrategias permiten que las decisiones tomadas sean en base a la visión, misión y objetivos planteados lo que permitirá la expansión a largo plazo de las mismas, esto demuestra que las estrategias son de gran importancia pues permiten el logro de los objetivos de las instituciones. 
Johnson y Scholes (2006) aseguran que una estrategia es considerada como un grupo de acciones llevadas a cabo con el fin de lograr un resultado especifico, se basa en la selección de un proceso determinado mediante el cual se espera la consecución de objetivos, habitualmente el concepto de estrategia es utilizado de tres formas, en primer lugar se la utiliza para poder determinar los medios que permitirán la consecución de un objetivo, también puede ser utilizada para establecer la forma de actuación a fin de lograr ventajas sobre el resto y finalmente también puede utilizarse para determinar los procedimientos o medios destinados que emplearán en caso de suscitarse una situación de confrontación a fin de lograr un objetivo.

La Constitución de la República Bolivariana de Venezuela (como se citó en Armas y Colmenares de Eizaga, 2007) asume que la recaudación optima de los tributos nacionales deben ser a través de un moderno sistema integral de Administración Tributaria con bases en la eficiencia, confianza y respeto hacia los contribuyentes, esto debe procurar que la distribución de las obligaciones publicas sean de acuerdo con la capacidad económica que cada contribuyente genera, todo esto debe estar motivado en el principio de progresividad, en este caso el empleo de la informática dentro de las administraciones tributarias pues permite mejorar la gestión de procesos de control y también proponer el ágil cumplimiento y conocimiento de las obligaciones y derechos de los deberes de la ciudadanía.

En el Ecuador según la nueva visión del SRI los contribuyentes son los principales evasores por lo que se estimarán los riesgos en los que se incurre por el incumplimiento de las obligaciones tributarias para ello se debe: a) rediseñar las estructuras de los procesos de auditoria, b) realizar modificaciones en la normativa que procure incrementar el control, (c) considerar dentro de los normativa enunciados sobre fiscalidad internacional, y (d) determinación de las fórmulas de cálculo del anticipo del impuesto a la renta y otros beneficios e incentivos tributarios enmarcados en la normativa (Roca, 2009).

A nivel mundial se desarrollan estrategias generalmente reconocidas que se adoptan en la gran mayoría de países como acciones que impulsan la recaudación y control fiscal por lo que Cortázar (como se citó en Carly, 2011) identifica tres estrategias que permiten fomentar la gestión de las administraciones tributarias, las mismas que están relacionadas con: a) gestiones publicitarias sobre los valores que motiven a la comunidad al cumplimiento de las obligaciones tributarias, b) acciones que fomenten la cultura a través de valores que se impartan a la ciudadanía a nivel escolar y c) gestiones que motiven la conciencia tributaria a nivel general, basándose en impartir o inculcar la importancia que tienen los tributos para el desarrollo general de la población.

Noya et al. (2014) señalan que para desarrollar estrategias de control fiscal es fundamental identificar los principales elementos del orden tributario de un país, y dentro de estos se encuentran: la estructura tributaria y la presión fiscal, a lo que se determina que la estructura tributaria es un indicador que permite diagnosticar los elementos y particularidades de las 
economías de los países y por lo tanto al financiamiento del Estado, en lo que respecta a la presión fiscal, existen múltiples estudios que vinculan los niveles de desarrollo de los países con las tasas de presión fiscal como porcentaje del PIB de los países, es decir, esto permite establecer una relación entre la proporción de impuestos con respecto del total de la producción de un país.

Dentro de las investigaciones citadas por los autores se encuentra un estudio realizado por el Instituto Argentino de Análisis Fiscal en el año 2012 en el que se evidencia que la importancia referente a la recaudación que se encuentra ligada con la presión y estructura tributaria de varios países; como por ejemplo en los EEUU el 64,23\% de los impuestos tienen como fuente el impuestos sobre la renta; mientras que países latinoamericanos este impuesto no supera el $40 \%$ de la recaudación sino más se enfocan en la recaudación de impuestos relacionados al consumo (ibídem).

El control fiscal por parte de las Administraciones Tributarias se basa en la denominada política de control fiscal que se encarga de proteger los intereses tributarios del Estado, buscando la determinación de los delitos tributarios, dentro de los principales objetivos de esta política es la implementación de estrategias para la recuperación de tributos por parte de productos que pudieran salir de del territorio, tratando de poner un alto a conductas ilegales para lo que propone acciones correctivas y de restricción en los controles ejecutados (Ministerio de Hacienda de Costa Rica, 2016).

La Comisión Económica para América Latina y el Caribe CEPAL, NU. (2017) establece que a partir del año 2016 en América Latina se han implementado una serie de estrategias que incentiven el cumplimiento tributario tanto a nivel nacional como internacional, se puede evidenciar un sin número de medidas entre las cuales se vieron en la necesidad de regular principalmente los temas relacionados con la elusión, precios de transferencia, la doble imposición mediante convenios internacionales, así como también la modificación de algunas reformas, mismas que son presentadas en la siguiente tabla:

Tabla 1. Matriz de estrategias de control fiscal en América Latina

\begin{tabular}{ll}
\hline País & Estrategias \\
\hline Ecuador & La promulgación de lineamientos para la correcta determinación de una jurisdicción de una \\
& menor imposición o paraíso fiscal. \\
& Establecer reformas a la Ley de Régimen Tributario Interno que permitan a la \\
& Administración Tributaria evidenciar la información de empresas vinculadas a los paraísos \\
& fiscales. \\
& Solicitar a todos los asesores y empresas de consultoría la información relacionada a los \\
& paraísos fiscales. \\
\hline Brasil & Establecer criterios que permitan afirmar la actividad económica real que desarrolla una \\
& empresa dentro de la economía del país. \\
& El desarrollo de un programa que regule los activos no declarados en el exterior. \\
\hline
\end{tabular}




\begin{tabular}{ll}
\hline Perú & Creación de un régimen de declaración para la repatriación de las rentas que no han sido \\
& declaradas. \\
\hline Uruguay & Se eliminó el sigilo bancario y se estableció a las instituciones financiera la obligatoriedad \\
& anual de reportar ante la Administración Tributaria los valores y saldos de las cuentas que \\
& poseen tanto las personas naturales como jurídicas con o sin residencia fiscal en el país. \\
\hline Bolivia & Se creó el Sistema de Facturación Virtual (SFV) de manera obligatoria para un tipo de \\
& contribuyentes, así como el desarrollo de aplicativos que permitan el cumplimiento de las \\
& obligaciones tributarias como por ejemplo el aplicativo QRquincho que se encarga de la \\
& lectura de los códigos QR que permite generar registros compatibles con el cierto tipo de \\
& formulario. \\
\hline & La Agencia Estatal de Administración Tributaria (AEAT) ha desarrollado estrategias desde \\
& el año 2012 enfocados en incentivar una mejor relación con los contribuyentes para motivar \\
& el cumplimiento de las obligaciones logrando una estrecha relación con el Estado, por lo \\
& que la AEAT considera importante la educación fiscal para quienes realizan actividades \\
& económicas que gravan impuestos, pero también se considera de gran importancia impartir \\
& la educación tributaria a los jóvenes a través de inculcarles valores, respeto y conciencia \\
& sobre el cumplimiento de sus tributos enmarcados en la normativa vigente.
\end{tabular}

Fuente: Ruiz de Zuazu (2014)

\section{Mecanismos de recaudación del impuesto a la renta en los sistemas tributarios}

En Chile la regla general es que los impuestos sean sujetos a Declaración, lo que constituye la base inmediata para la determinación y pago del impuesto. Tanto el Impuesto a la Renta como el IVA son impuestos sujetos a declaración, de manera que es el propio contribuyente quien debe declarar que ha obtenido una renta, cuál es el monto de ésta y finalmente establecer la cantidad que le corresponde pagar por concepto de impuestos. La Administración Tributaria únicamente efectuará la determinación del impuesto o de una diferencia de los mismos cuando, a raíz de un procedimiento de fiscalización, detecte que el contribuyente no declaró, o bien, que su declaración es errónea o falsa (Centro Interamericano de Administraciones Tributarias, 2008).

El Estado mexicano se basa en varios principios, según el principio de economía citado por Adam Smith en 1776 en lo que respecta a recaudación de impuestos, busca establecer diferencias entre lo recaudado por la Administración y lo que efectivamente pago el contribuyente, a través de la implementación de medios de control y cobro de impuestos sencillos y poco costosos, uno de ellos es la implementación servicios tecnológicos para ayudar a los contribuyentes logrando de esta manera reducir los gastos en la recaudación de los ingresos para el Estado (Sol, 2012). 
El impuesto a la renta en algunos países constituye el pilar principal del sistema tributario debido a que es el impuesto más distributivo y sobre el cual más recaudación se obtiene con relación a otros impuestos, en la mayoría de los países europeos uno de los métodos utilizados es el impuesto a la renta lineal que consiste en aplicar la misma tasa impositiva a las todas las fuentes y cuantías de ingreso recibidas tanto para las personas naturales como jurídicas este método se basa en principio de progresividad y se logra al establecer un mínimo exento del tributo determinado, dicho métodos surge como necesidad de implementar una forma sencilla para la recaudación de los impuestos en aquellas administraciones tributarias que son menos experimentadas (Corbacho, Fretes, y Lora 2012).

Es imprescindible que todos los Gobiernos tengan los ingresos suficientes para poder hacer frente a la financiación de sus operaciones relacionadas con servicios, infraestructura y desarrollo económico de la población, por lo que la principal misión de las administraciones tributarias es el de recaudar ingresos fiscales regidos mediante leyes tributarias de cada país y que son primordiales para el desarrollo del sector, para lo que deben enfrentarse a varios desafíos en la gestión de procesos que les permitan el cumplimiento voluntario y el pago de los impuestos mediante el cumplimiento de sus responsabilidades que se basan principalmente en la administración de las leyes tributarias, la minimización de costos y cargas para los contribuyentes, el constante monitoreo de las obligaciones, propagar el desarrollo eficiente por parte de los funcionarios, entre otras. Así mismo la Administración Tributaria debe reflejar un alto grado de eficiencia y eficacia en el diseño de sus sistemas para el control y recaudación de los procesos que tienden a presentar un alto riesgo de cumplimiento de los contribuyentes y por lo tanto su impacto afectará de manera significativa en los ingresos para los gobiernos (Jacobs, 2013).

Son innumerables los mecanismos que se utilizan en diferentes países para la recaudación del impuesto por lo que se constituye como una de las principales preocupaciones de la Administración Tributaria, ya que lo que se busca es recibir la totalidad de la recaudación de los tributos y que sean incluidos en las arcas fiscales, por lo se implementan medidas de control para los procesos tributarios, según lo que establece el autor Jacobs (2013) sobre los mecanismos utilizados para la recaudación de impuestos establece que las administraciones tributarias deben procurar recibir ingresos derivados de las cargas tributarias impuestas a los contribuyentes pero en base a programas eficientes que logren ejercer presión pero que a su vez motiven a la ciudadanía al cumplimiento voluntario de sus obligaciones ya que esto incidirá en los fondos recibidos por el Estado y a sus vez afectará directamente a los planes de desarrollo social que se puedan implementar en el país.

El control tributario de la evasión y elusión del sistema tributario con un enfoque Nacional.

Se considera como delito de defraudación tributaria a la evasión fiscal pues tiene relación por un lado con la disminución de los ingresos y por otro con la excesiva deducción de gastos, con el único objetivo de evitar el pago de los impuestos por parte del sujeto deudor del tributo, 
además ocasiona perjuicios para el Estado llegándose a recaudar pocos ingresos lo que disminuye recursos para distribuir al Presupuesto General, además ante la constante necesidad de incrementar la recaudación puede ocasionar la creación de nuevos impuestos o incluso el incremento de las tasas impositivas, por otro lado provoca una desigualdad en la carga tributaria pues ocasiona una reducción del número de contribuyentes sobre los cuales se redistribuyen los impuestos (Corporación de estudios y publicaciones CEP, 2011).

La evasión corresponde a todo hecho de incumplimiento de la ley que ayuda a los contribuyentes a evitar sea de manera total o parcial el pago de los impuestos, este comportamiento por parte de quienes están en la obligación de cumplir con dicha imposición afecta de manera directa a los ingresos percibidos por el Estado perjudicando al sistema tributario en términos de recaudación, pues tiene como consecuencias la violación a la norma y reglamentos de ley a fin de disminuir la base imponible de los ingresos sobre los cuales se debe contribuir (Andino, 2012). Carpio (2012) refiere que la evasión se origina luego del nacimiento de la obligación tributaria en el cual el contribuyente realiza algún tipo de engaño que impide que la Administración Tributaria pueda tener pleno conocimiento del valor real sobre el cual debe calcular el hecho generador del impuesto, produciendo una violación de la ley.

Torres (2013) cita que la elusión tributaria es el acto que da lugar a la reducción de la base imponible a través de establecer acciones que permite reducir la base imponible mediante procedimientos que no están prohibidos por la normativa tributaria, lo que es utilizado por el deudor de un tributo para minimizar su imposición tributaria haciendo uso de la ley.

Mayorga (2017) señala que la elusión es todo acto basado en vacíos legales en donde se buscar la interpretación de la norma a favor del contribuyente, el principal objetivo que busca la elusión es disminuir del pago de un impuesto ya generado por al sujeto pasivo, generalmente se amparan en aspectos que el ente de control no considero dentro de las regulaciones de la base legal.

La elusión es habilidad que tiene el contribuyente para diseñar o utilizar varios sustentos jurídicos para realizar de alguna manera un fraude a la ley con el objetivo de mermar la base imponible real sobre la cual se deriva el pago de una obligación tributaria, Es importante indicar la elusión es diferente a la evasión porque no se incumple con la presentación de la obligación sino más bien se trata de reducir la base imponible sobre la cual se debe declarar (Matus, 2017).

\section{Diferencias entre evasión y elusión tributaria}

Las principales diferencias establecidas se muestran en la figura 2. 
Figura 2. Diferencias básicas entre evasión y elusión tributaria.

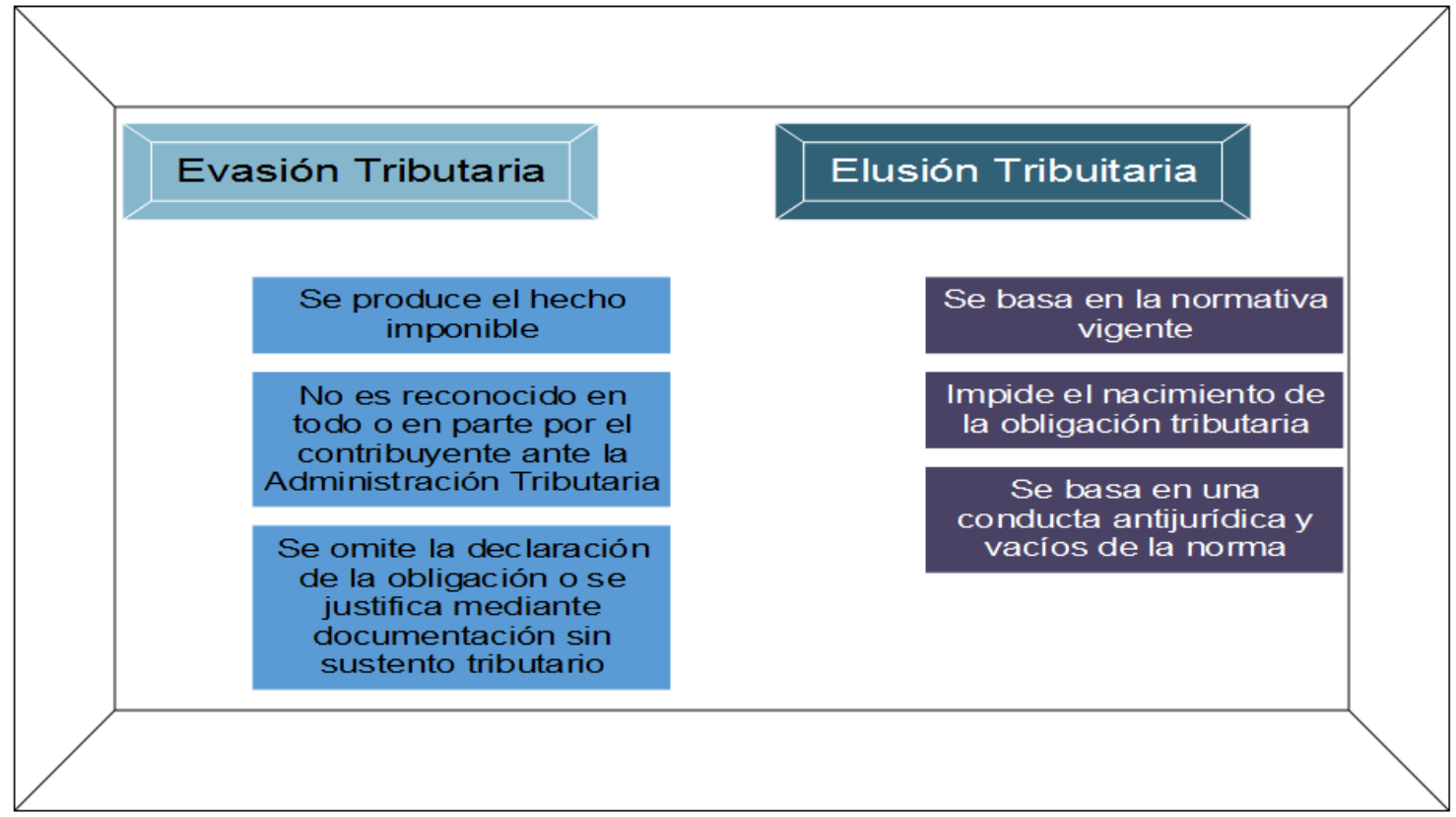

Fuente: Elaboracion propia

\section{Causas de la evasión y elusión tributaria.}

Fatás y Roig (2004) refieren que la evasión es considerada como una actividad ilegal que al querer ser erradicada se debe considerar que está ligada a dos conductas, por un lado a los graves problemas de las organizaciones que están relacionados a la intervención directa de su personal y por otro lado su generalización lo que hace que se penalice a todos los contribuyentes incluyendo a quienes no infringen la ley y esto a su vez provoca la disminución de la credibilidad de los sistemas tributarios.

Generalmente los contribuyentes que evaden tributos se enmarcan en realizar acciones evasoras como: a) no cumplir con sus declaraciones, y b) considerar rubros o incluso valores inexistentes que les permitan generen un saldo a su favor a fin de evitar ser sancionados por la administración, este comportamiento se base en el criterio de que la existencia de la evasión en la sociedad induce al resto de contribuyentes a su cometimiento a fin de procurar su permanencia en el mercado, así como también la visión del alto gasto de recursos por parte del Estado e inclusive aun cuando se incrementan las tarifas impositivas y finalmente que dentro de norma se pueda contemplar algunos vacíos sobre los que se pueda justificar dicha evasión (Macías, Agudelo y López, 2007). 
El incumplimiento de las obligaciones tributarias a través de la evasión y elusión es causada por diferentes factores entre los que se destacan: la compleja y débil estructura legal del tributo que en su mayoría está relacionado a las constantes actualizaciones de la normativa, la falta de mecanismos de control eficientes que disminuyan los riesgos de detección de incumplimiento, el constante abuso de los beneficios tributarios establecidas para ciertos sectores, la actitud reacia de los contribuyentes al pago de los impuestos a pesar de las acciones de cobro adoptadas por el Estado y la falta de conocimiento de las disposiciones tributarias que incluso están relacionadas con la desigualdad económica (Comisión Económica para América Latina y el Caribe CEPAL, 2010).

Por otro lado, es importante mencionar que la evasión por la falta de cumplimiento de las obligaciones tributarias afecta de manera considerable la recaudación, llegando a alterar el impacto distributivo de la riqueza, situación que puede afectar tanto la equidad horizontal pues la evasión llegaría a permitir que dichos evasores acaben contribuyendo en menor grado que quienes cumplen con sus obligaciones así como afectar a la equidad vertical puesto que quienes poseen más capacidad contributiva tienen más acceso a asesorías para aplicar estrategias que le permitan la evasión o elusión tributaria (Sanguinetti, et al., 2012).

Domínguez (2013) afirma que una de las principales causas que generan incumplimiento y evasión fiscal es la falta de ética de la ciudadanía lo que debilita e impide los controles por parte de la Administración Tributaria, debiendo considerar que se requiere alcanzar un equilibrio entre la concientización de los deberes y derechos de los contribuyentes y los procesos de control que desarrolla el Fisco.

\section{Procesos de control de omisidad empleados por las administraciones tributarias.}

Los procesos de control de omisidad establecidos por el Servicio de Rentas Internas en el Ecuador está enmarcado hacia la verificación del cumplimiento de las obligaciones dentro de los plazos de ley por parte del sujeto pasivo, para ello las principales funciones que ejerce son las de persuadir y sancionar amparado en la base legal, cabe indicar que lo que la administración busca es reducir tanto las brechas de cumplimiento de la obligación y veracidad de la información, dichos controles se realizan en base a la planificación oportuna de controles y también en base a la disponibilidad de personal recursos (Coronel, 2014).

Durango (2017) indica que los procesos de control de omisidad buscan cerrar la brecha de presentación de las declaraciones por parte de los sujetos pasivos del impuesto, pero se debe tener presente la materialidad que repercute la realización de un control a determinado grupo de contribuyentes, para ello la Administración Tributaria deberá considerar el impacto que causará el control de dicho impuestos sobre los ingresos que espera recaudar el Estado, además si bien se debe considerar los controles bajo el principio de generalidad buscando que los contribuyentes registrados cumplan con sus obligaciones tributarias, también se debe 
dar prioridad de control a aquellos sujetos pasivos que con el cumplimiento de sus declaraciones nos van a repercutir mayores recursos.

Los cumplimientos de las obligaciones tributarias por parte de los contribuyentes dependen de algunos factores tales como: a) La moral, que en su gran parte se deriva del pensamiento social que puedan tener los contribuyentes; b) la posición que tenga el ente de control ante los sujetos pasivos y c) el conocimiento de la sociedad sobre la estructura e imposición de los tributos (Comín, 2018).

\section{Metodología.}

El enfoque del presente estudio fue mix to ya que mediante el método cualitativo se analizó una realidad del proceso efectivo de recaudación del anticipo bajo la normativa tributaria vigente y mediante el método cuantitativo se hizo uso de información estadística en base a una encuesta sobre los procesos de recaudación del anticipo del impuesto a la renta.

El alcance de la investigación fue descriptivo ya que se fundamentó teóricamente las variables de estudio, en cuanto a la finalidad la investigación tuvo un horizonte transversal ya que la toma de información de los procesos de control de omisidad de las obligaciones tributarias y datos de la recaudación del anticipo del impuesto a la renta se realizó en un tiempo específico. Partiendo de lo que señala Rodríguez y Pérez (2017) la presente investigación se fundamentó en el método histórico pues se realizó un estudio bibliográfico de las leyes, normativas y reformas tributarias de la legislación ecuatoriana considerando las modificaciones del cálculo del anticipo del impuesto a la renta y posteriormente se realizó las debidas comparaciones de las variables investigadas. Además, se utilizó el método inductivo-deductivo, el mismo que partió de conceptos generales sobre los procesos de control de omisidad para luego determinar la relación que existe particularmente con las estrategias para la recaudación. Las técnicas de investigaciones empleadas fueron la encuesta, la entrevista y el focus group.

El presente estudio de investigación se realizó mediante la participación voluntaria de 15 funcionarios del área de control del Servicio de Rentas Internas a nivel Nacional, así también se utilizó material tributario que consta en la página Web de dominio público de la institución. 


\section{Resultados.}

De la fase de diagnóstico ejecutada se obtuvieron los siguientes resultados:

El $67 \%$ de los encuestados concluye que existe un elevado número de contribuyentes que no tienen acceso a la información oportuna de las reformas tributarias relacionadas a la determinación y pago del anticipo del impuesto a la renta, ya sea por propio desinterés del contribuyente o por indisponibilidad de los sistemas digitales, esto conlleva a que no exista el debido cumplimiento de la obligación tributaria.

Los contribuyentes no tienen un conocimiento de los rubros que se consideran para determinar el anticipo del impuesto a la renta, así como no utilizan de forma correcta las exenciones por concepto de anticipo del impuesto a la renta, lo que ocasiona que los contribuyentes frecuentemente hagan una determinación equivocada de su anticipo ya que en los procesos de control mediante cruces de información se puede evidenciar que existen diferencias entre la declaración del contribuyente y la información reportada por terceros.

Se determinó, además que una gran parte de los funcionarios consideran que las instituciones financieras juegan un papel fundamental pues la información de los estados financieros de los contribuyentes que los bancos poseen es un factor determinante para la Administración Tributaria al momento de validar y certificar que los movimientos económicos del contribuyente sean los reales y de esta manera poder realizar un proceso de control sobre información inequívoca.

No se debería considerar la información del ejercicio impositivo anterior para determinar el pago del anticipo del impuesto a la renta del año en curso, pues se estaría pagando un anticipo bajo supuestos de que los contribuyentes van a mantener o incrementar su situación económica, este método de cálculo tiende a incrementar la carga impositiva de los contribuyentes disminuyendo su liquidez en las fechas que deben realizar los pagos de este impuesto.

Se evidencio que al existir varios escenarios para el cálculo del anticipo del impuesto a la renta que están ligados al tipo de contribuyente y la actividad económica que realizan, se dificulta el proceso de control de diferencias por anticipo del impuesto a la renta, pues en la actualidad el control se basa únicamente en la verificación de la correcta aplicación de la formula debido a que por cuestiones de tiempo y de carga operativa se limita el control en la determinación de los rubros que se contemplan para el cálculo del impuesto. 
En lo relacionado al anticipo del impuesto a la renta se puede evidenciar que el $40 \%$ de los encuestados indican que se debe considerar la eliminación de exenciones a fin de evitar la mala interpretación por parte de los contribuyentes mientras que el otro $60 \%$ considera que se debe evaluar y conservar cierto tipo de exenciones siempre que incentiven la productividad de las empresas y que a su vez no representen un alto grado de disminución en la recaudación por concepto de anticipo del impuesto a la renta.

\section{Propuesta de estrategias para la recaudación del anticipo del impuesto a la renta en el Ecuador}

Luego de los resultados obtenidos mediante la aplicación de las técnicas de investigación, se propone implementar las siguientes estrategias:

Implementar procesos de capacitación continua sobre la forma de determinación del anticipo del impuesto a la renta en base a la normativa vigente que permita efectivizar la recaudación del anticipo minimizando los excesivos procesos de control pues los contribuyentes actualmente presentan cierto tipo de sigilo ante la Administración Tributaria, logrando así, que los contribuyentes consideren necesario realizar una planificación tributaria que permita efectivizar los métodos y formas de cálculo del anticipo sin necesidad de afectar los presupuestos del contribuyente.

Por otro lado, se considera importante disponer y utilizar la información de los balances y estados financieros presentados antes las instituciones bancarias, con el objetivo de poseer información real de la situación económica de los sujetos pasivos y que esto permita validar la información declarada por los contribuyentes ante el Servicio de Rentas Internas y la información que se presenta ante los bancos.

La modificaciones a la normativa correspondiente con el cálculo del anticipo se deberá sustentar en el análisis de incentivos y exenciones a fin de que sean focalizados hacia dos conceptos principales: a) el incremento de la inversión en activos relacionados directamente a la actividad económica de los contribuyentes que permita mejorar la productividad de su negocio; y, b) la generación de nuevo empleo a través de la exención de un determinado porcentaje de costos y gastos en que se incurran para la generación del empleo, todo esto encaminado bajo el concepto de simplicidad administrativa, los otros tipos de exenciones o exoneraciones tienden a ser punto frágil para la evasión y elusión de impuestos por lo que se debe considerar su eliminación. 
Aplicar una nueva fórmula de cálculo del anticipo del impuesto a la renta basados en la adaptación de la normativa tributaria internacional, en la que hace referencia a que el anticipo se determina mediante un porcentaje que en materia tributaria puede ser aplicado a la utilidad gravable producto del ejercicio económico real, menos las retenciones del impuesto a la renta y la participación de trabajadores, este método de cálculo debe ser considerado para las sociedades, las sucesiones indivisas y las personas naturales obligadas y no obligadas a llevar contabilidad, basados en los principios de igualdad y equidad tributaria, sin dejar de contemplar la posibilidad de que si el contribuyente demostraré ante la Administración Tributaria que tuvo pérdidas en el ejercicio fiscal pueda solicitar la devolución del valor cancelado por anticipo y que este proceso de devolución sea automático una vez que se valide la perdida fiscal por parte del departamento de control.

Es importante que la Administración Tributaria desarrolle planes de control periódicos y que como resultado de esto se identifique en una base de datos a los contribuyentes que frecuentemente tienen problemas para el cálculo y determinación del anticipo del impuesto a la renta, a los cuales deberá brindar una capacitación focalizada que permita mitigar dichas confusiones, los controles que se desarrollen deberán detallar las exoneraciones y otros beneficios de ley de los que haya hecho uso el contribuyente; y también especificar los rubros que se contemplen dentro del cálculo del anticipo, para ello la administración tributaria deberá establecer un cronograma para controles dependiendo de la materialidad de la recaudación y en base al catastro de contribuyentes.

Es importante establecer sanciones rígidas para los contribuyentes que hacen mal uso de las exenciones, exoneraciones y beneficios tributarios por concepto de anticipo del impuesto a la renta, incluso contemplar la posibilidad de una sanción que le prohíba de manera indefinida el uso de los mismos como castigo a sus malas prácticas tributarias.

\section{Conclusiones.}

Para el desarrollo de estrategias de recaudación del anticipo, el organismo de control deberá analizar la normativa tanto nacional como internacional, para en base a ello poder identificar las principales falencias que presenta el sistema tributario nacional, permitiendo implementar reformas que incrementen los ingresos del Estado.

Con las reformas vigentes se pudo lograr que varios contribuyentes que no venían declarando

por concepto del anticipo del impuesto a la renta actualmente realicen su cálculo de manera correcta, disminuyendo la probabilidad de la manipulación de la información para declarar 
perdidas y no pagar anticipo que es de cierta forma una recaudación anticipada del impuesto a la renta.

La mayor parte de los contribuyentes consideran que el pago del anticipo limita su capacidad de reinversión y las oportunidades de inversión extranjera, por lo que es importante concientizar a los contribuyentes sobre la importancia de una adecuada cultura tributaria.

\section{Referencias bibliográficas.}

Andino, M. (11 de 2012). Instrumentos y Técnicas para medir la Evasión. Obtenido de https://www.taxcompact.net/documents/seminar-montevideo2/2012-1122_itc_Andino_SRI.pdf

Andrade, M. S. (06 Bde 2013). Factores asociados a la impugnación de las obligaciones tributarias y su efecto en la recaudación fiscal ecuatoriana. Tesis de Maestría, 70. Quito, Ecuador: FLACSO Sede Ecuador. Obtenido de http://hdl.handle.net/10469/5899

Arias, D., y Cano, L. (12 de 2009). Benchmarking de Imposición Directa en Iberoamérica 2009. Documento de Trabajo No. 2009-04. Quito, Ecuador: Centro de Estudios Fiscales. Obtenido de https://cef.sri.gob.ec/pluginfile.php/16783/mod_page/content/138/2009_04.pdf

Armas A, M., y Colmenares de Eizaga, M. (2007). Las nuevas tecnologías en las administraciones tributarias. Red de Revistas Científicas de América Latina y el Caribe, España $\quad$ y $\quad$ Portugal. Obtenido de http://www.redalyc.org/articulo.oa?id=78460306

Asamblea Nacional Constituyente (2016). Código Tributario del Ecuador. Quito: Ediciones Jurídica del Ecuador.

Asamblea Nacional Constituyente (2007) Reglamento para la aplicación de la Ley de régimen tributario interno. Quito: Ediciones legales.

Asamblea Nacional Constituyente. (2015). Ley reformatoria para la equidad tributaria en el Ecuador. Quito: Ediciones Legales.

Asamblea Nacional Constituyente. (2017). Ley de Régimen Tributario Interno. Quito : Ediciones legales. 
Benítez, C. M. (2008). Introducción a la Tributación. Loja: Universidad Técnica Particular de Loja.

Carly, A. S. (03 de 2011). Sistema de Recaudación Tributaria en Materia de Impuestos sobre las Actividades Económicas de Industria, Comercio, Servicio e índole similar: (Caso: Dirección de Hacienda de la Alcaldía del Municipio Colón del Estado Zulia). Tesis de Postgrado. Mérida, Yucatan, México.

Carpio, R. R. (2012). La planificación tributaria internacional / The international tax planning. Revista Retos, 53-67. Obtenido de http://dspace.ups.edu.ec/handle/123456789/8074

Centro de Estudios Fiscales. (02 de 2017). Incidencia de las propuestas tributariasen el periodo electoral 2016 - 2017. Documento de Trabajo No. 2017-01. Quito, Ecuador. Obtenido de https://cef.sri.gob.ec/pluginfile.php/48061/mod_page/content/3/201701.pdf

Centro Interamericano de Administraciones Tributarias. (11 de 2008). Evasión Tributaria. Serie temática tributaria CIAT. Servicio de Impuestos Internos - SII. Obtenido de http://www2.congreso.gob.pe/sicr/cendocbib/con4_uibd.nsf/0872CF4513D1870F05 257C1300032C14/\$FILE/2008_nov_n2_evasion.pdf

Chuquimarca, c. J. (12 de 2013). Variables del concepto de renta en los países miembros de la CAN. Tesis de Maestría. Quito, Ecuador. Obtenido de http://hdl.handle.net/10644/3904

Comín, F. (2018). La corrupción permanente: El fraude fiscal en España. Revista de Historia Contemporánea, 481-521. doi: https://doi.org/10.20318/hn.2018.4046

Comisión Económica para América Latina y el Caribe CEPAL. (2010). Evasión y equidad en América Latina. Santiago de Chile, Chile: Naciones Unidas. Obtenido de https://repositorio.cepal.org/handle/11362/3762

Comisión Económica para América Latina y el Caribe CEPAL, NU. (2017). Panorama Fiscal de América Latina y el Caribe 2017: la movilización de recursos para el financiamiento del desarrollo sostenible. Santiago CEPAL. Obtenido de http://hdl.handle.net/11362/41044

Corbacho, A., Fretes, V. y Lora, E. (2012). Recaudar no basta, los impuestos como instrumento de desarrollo. (B. I. Desarrollo, Ed.) Obtenido de 
http://www.aecid.es/Centro-

Documentacion/Documentos/documentos\%20adjuntos/Fiscal\%20BID.pdf

Coronel, R. (03 de 2014). Segmentación de contribuyentes y control de omisos: diagnóstico y recomendaciones". Tésis de Maestría. Quito, Ecuador. Obtenido de http://repositorio.iaen.edu.ec/handle/24000/3960

Corporación de estudios y publicaciones CEP. (2011). El Ilícito tributario . Quito : Ecuador.

Delgado, F. y Nuñiz, M. (2005). Cuadernos de hacienda pública teoría de los impuestos. Oviedo: Universidad de Oviedo.

Domínguez, G. M. (2013). Fiscalidad, su cumplimiento estrechamente ligado al concepto de ciudadanía. En P. d. contribuyente, Retos y Perspectivas para una Nueva Cultura Contributiva en México (pág. 192). México.

Durango, C. (2017). Brecha de presentación de declaraciones en la provincia del Azuay, herramientas para el control de omisos. Propuesta de estrategia para el control de la brecha de presentación. Tésis de Maestría. Cuenca, Ecuador. Obtenido de http://dspace.ucuenca.edu.ec/handle/123456789/27088

Fatás, E. y Roig, J. (2004). Equidad y evasión fiscal. un test experimental. Revista de Economía Aplicada, XII (34), 17-37. Obtenido de http://www.redalyc.org/articulo.oa?id=96917645002

González, E. y Lejeune, E. (2003). Derecho Tributario. Salamanca: Plaza Universitaria. Obtenido de https://www.popularlibros.com/libro/derecho-tributario-i_86105

Hernández, G. F. (2016). La justicia tributaria en el ordenamiento plural. Revista Boliviana de Derecho, 156-173. Obtenido de http://www.redalyc.org/articulo.oa

Jacobs, A. (2013). Directrices Detalladas para la Mejora de la Administración Tributaria en América Latina y el Caribe. Agencia Internacional para el Desarrollo de los Estados Unidos. Deloitte Consulting LLP. Obtenido de https://www.usaid.gov/sites/default/files/LAC_TaxBook_Ch\%202\%20$\%$ 20SPANISH.pdf

Johnson, G. y Scholes, K. (2006). Dirección Estratégica (Vol. $7^{a}$ Edición ). Madrir: Prentice Hall. 
Loyo, G. F. (2003). La evasión de los impuestos al comercio exterior. Tesis de maestría. Obtenido de http://repositorio.uasb.edu.ec/bitstream/10644/2459/1/T0266-MDELoyo-La\%20evasi\%C3\%B3n.pdf

Macías, H., Agudelo, L., y López, M. (2007). Los métodos para medir la avasión de impuestos: una revisión. Semestre Económico, 67-85. Obtenido de http://www.redalyc.org/articulo.oa?id=165013675004

Matus, M. (2017). La elusión tributaria y su sanción en la Ley $\mathrm{N}^{\circ} 20.780$. Hacia un concepto de negocio. Ius et Praxis, 67-89. Obtenido de ttp://www.redalyc.org/articulo.oa?id=19752296003

Mayorga, I. (2017). Sociedades instrumentales y su vinculación con los paraísos fiscales, como elemento de elusión y evasión para el pago de impuesto a la renta en el caso ecuatoriano. Tesis de maestría. Quito. Obtenido de http://hdl.handle.net/10644/5416

Mejía, A. J. (2015). Análisis jurídico del anticipo del Impuesto a la Renta en el Ecuador. Tesis de Maestría. Quito, Ecuador. Obtenido de http://repositorio.uasb.edu.ec/bitstream/10644/4666/1/T1724-MDE

Ministerio de Hacienda de Costa Rica. (2016). Guía para educación superior Estado, tributación y ciudadanía. Costa Rica: Subdirección de Educación y Cultura Fiscal, Dirección General de Tributación, Ministerio de Hacienda de Costa Rica. Obtenido de http://educa.hacienda.go.cr:8080/costarica_prod/

Noya, N., Fernández, N., Andrada,D., Gerez,L., González, D., y Ricotta, N. (2014). Finanzas públicas. Buenos Aires: Editorial de la Universidad Nacional del Comahue. Obtenido de http://www.eumed.net/libros-gratis/2016/1503/presion-fiscal.htm

Piffano, H. L. (2012). Análisis económico del derecho tributario. La Plata: Universidad Nacional de la Plata. Obtenido de https://libros.unlp.edu.ar/index.php/unlp/catalog/view/254/231/756-1

Procuraduría de la Defensa del Contribuyente. (2015). Cultura contributiva en América Latina. México: Procuraduría de la Defensa del Contribuyente PRODECOM. Obtenido de http://www.prodecon.gob.mx/index.php/home/cc/publicaciones/culturacontributiva-en-america-latina-2015 
Ramírez, J., Cano, L. y Oliva, N. (12 de 2009). Impuesto a la Renta de Personas Naturales en Relación de Dependencia Un Análisis de Equidad y Redistribución. Documento de Trabajo No. 2010-01. Quito, Ecuador. Obtenido de https://cef.sri.gob.ec/pluginfile.php/16803/mod_page/content/15/2010-01.pdf

Robbins, S. y Coulter, M. (2005). Administración. México, México: PEARSON EDUCACIÓN. Obtenido de http://www.cars59.com/wpcontent/uploads/2016/02/Book-Administracion.pdf

Roca, G. (09 de 2009). Tributación directa en Ecuador: evasión, equidad y desafíos de diseño. Serie Macroeconomía del Desarrollo. CEPAL. Obtenido de https://repositorio.cepal.org/handle/11362/5456

Rodas, W. M. (2006). La gestión de los tributos y las garantías del debido proceso en el Ecuador. Tesis de Maestría. Azogues, Ecuador. Obtenido de http://hdl.handle.net/10644/776

Rondón, C. J. (2017). Glosario Aduanero Tributario. Oficina de Información. Venezuela. Obtenido de http://noticias.seniat.gob.ve/index.php/glosario-tributario

Ruiz de Zuazu, M. (2014). Medidas sociales para combatir el fraude fiscal en España. Documento de trabajo. Obtenido de https://portal.uah.es/portal/page/portal/epd2_asignaturas/asig360050/informacion_a cademica/371dcdd58cc5313331737c8c08bd6d6e.articulo.fraude.2014.pdf

Salazar, P. (2008). Impuesto a la renta. Loja, Ecuador: Universidad Técnica particular de Loja.

Sanguinetti, P., Berniell, L., Álvarez, F., Ortega, D., Arreaza, A., y Penfold, M. (2012). RED 2012: Finanzas públicas para el desarrollo. Fortaleciendo la conexión entre ingresos y gastos (Reporte de Economía y Desarrollo (RED)). Caracas: CAF. Retrieved from http://scioteca.caf.com/handle/123456789/169

Sol, J. H. (2012). Derecho Fiscal. México: RED TERCER MILENIO S.C. Obtenido de http://www.upg.mx/wp-content/uploads/2015/10/LIBRO-6-Derecho-Fiscal.pdf

Tanzi, V. y Zee, H. (2001). La política tributaria en los países en desarrollo. Fondo Monetario Internacional.

Obtenido

de https://www.imf.org/external/pubs/ft/issues/issues27/esl/issue27s.pdf 
Torres, B. (2 de 2013). Elaboración de un modelo de control en el SRI Santo Domingo de los Tsáchilas para disminuir la Evasión y Elusión del pago del impuesto a la propiedad de vehículos motorizados. Tesis de Maestría. Quevedo, Ecuador. Obtenido de http://dspace.uniandes.edu.ec/handle/123456789/2879

Torres, C. M. (2008). Estructura de los ingresos tributarios en el presupuesto general del Estado. Tesis de Maestría. Quito, Ecuador. Obtenido de http://hdl.handle.net/10644/378

Torrico, G. M. (12 de 2015). El sistema tributario en el modelo de Estado Autonómico Boliviano. Ciencia y Cultura, 219-234. Obtenido de http://www.redal

Troya, J. (2008). Control de las leyes tributarias y los efectos de su declaración de invalidez. Foro: revista de derecho. 9 (Tabla de Contenido), 63. Quito: Universidad Andina Simón Bolívar, Corporación Editora Nacional. Obtenido de http://hdl.handle.net/10644/1417

Vergara, B. M. (2011). Sistema tributario eficiente, pilar para el desarrollo. Revista Perspectiva, $20 . \quad$ Obtenido de http://investiga.ide.edu.ec/images/pdfs/revistajulio2011.pdf

Zambrano, P. E. (08 de 2014). Análisis del anticipo del impuesto a la renta y la afectación de la liquidez de los contribuyentes en el Ecuador 2010 - 2013. Tesis de Maestría. Guayaquil, Ecuador.

Obtenido de http://repositorio.ug.edu.ec/bitstream/redug/6447/1/TESIS\%20ELLANNY\%20ZA MBRANO\%20.pdf

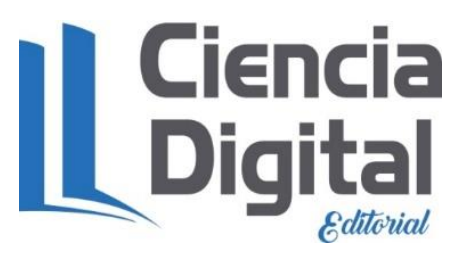


PARA CITAR EL ARTÍCULO INDEXADO.

González Valladares, C., Narváez Zurita, C., \& Erazo Álvarez, J. (2019). Estrategias para la recaudación del anticipo del impuesto a la renta en el Ecuador. Visionario Digital, 3(2.1.), 86-113. https://doi.org/10.33262/visionariodigital.v3i2.1.547

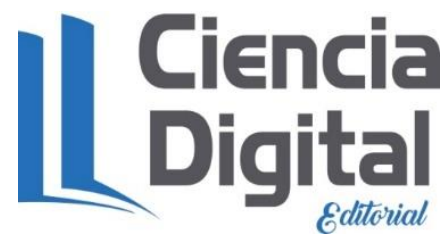

El artículo que se publica es de exclusiva responsabilidad de los autores y no necesariamente reflejan el pensamiento de la Revista Ciencia Digital.

El artículo queda en propiedad de la revista y, por tanto, su publicación parcial y/o total en otro medio tiene que ser autorizado por el director de la Revista Ciencia Digital.
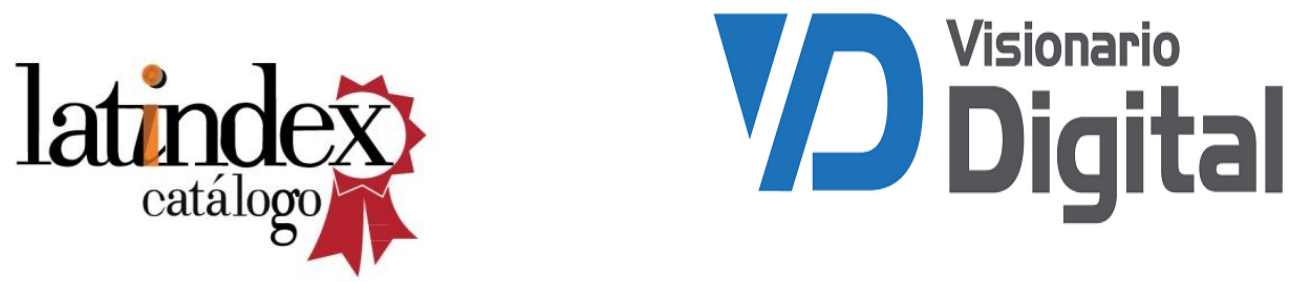